\title{
Mario Bunge y la estadística bayesiana
}

\author{
Mario Bunge and Bayesian statistics \\ Jorge Ortiz Pinilla国 \\ jeortizp@gmail.com
}

\section{Introducción}

Después de leer el artículo de Silva (2013), no quedan dudas sobre los errores técnicos y teóricos de Bunge (2003) cuando "demuestra" las contradicciones a las que se llega mediante el uso del teorema de Bayes. Tal vez, por su preparación matemática como físico, el filósofo no vio la necesidad de acudir a la revisión por parte de un probabilista o de un estadístico. La ingenuidad de sus errores y su lenguaje casi pasional deslucen la obra de un autor que se declara enemigo de la subjetividad.

Por otra parte, no se necesitan grandes teorías para deducir que es mejor saber algo que nada y que, cuando se conoce algo, es mejor aprovecharlo que no hacerlo. La fórmula de Bayes y todo lo que ha llevado a los desarrollos actuales de la estadística bayesiana simplemente ofrecen métodos para aprovechar el conocimiento adquirido. La sola presentación de los métodos debería bastar para convencer que lo hace bien; además, se dispone de una enorme y creciente cantidad de ejemplos de aplicaciones exitosas.

En lo que sigue, prefiero leer y tratar de interpretar al filósofo, sin la pretensión ( $a$ priori) de encontrar con ello motivación por el estudio de la estadística bayesiana.

\section{Probabilidades y proporciones}

El manejo y la interpretación de las probabilidades son realmente muy complejos. En eventos o experiencias repetibles, hace más de 300 años, Jacob Bernoulli encontró que la relación de la cantidad de éxitos sobre la de experiencias tiende a un valor igual al de la relación de casos favorables sobre casos posibles. La repetibilidad (en condiciones idénticas) abre un camino para acercarse al conocimiento de una relación $p$ inicialmente desconocida, pero fija.

\footnotetext{
${ }^{\text {a}}$ Facultad de Estadística, Universidad Santo Tomás (Colombia).
} 
¿Qué pasa si, como plantea Bunge (2012, p. 100), ya se ha probado que un cierto individuo $e$ tiene VIH y se pregunta por la probabilidad de que tenga sida? Para manejar el tema en un contexto de probabilidad, se necesita admitir que el individuo ya ha sido seleccionado de alguna manera que posibilita el uso de las probabilidades. Admitamos entonces que el espacio de probabilidad para la selección de los individuos está bien definido, que el evento $\{e\}$ forma parte de la $\sigma$-álgebra respectiva y que su probabilidad es un valor entre 0 y 1 . Supongamos ahora que el evento $\{e\}$ ha ocurrido y además ya se sabe que $e$ tiene VIH.

Llamemos $S E L$ al conjunto de seleccionados que, en este caso, consta de una sola persona. Como $e$ ya ha sido seleccionado y tiene $V I H$, entonces el evento $S E L \cap V I H=\{e\}$ ya ha sido observado. No hay riesgo de que su probabilidad sea cero. Entonces, la pregunta de Bunge se traduce en buscar $P(e \in \operatorname{sida} \mid\{e\})$. Por la definición de probabilidad condicional,

$$
\operatorname{Pr}(e \in \operatorname{sida} \mid\{e\})=\frac{P(e \in \operatorname{sida} \cap\{e\})}{P(\{e\})}
$$

Si $e$ tiene sida, el numerador en (1) es igual al denominador y la probabilidad buscada será igual a 1; en caso contrario, será cero. Como puede verse, este resultado es inútil, pues exige el conocimiento de la condición que precisamente se desconoce. El cálculo de probabilidades no da respuestas útiles para casos individuales ya determinados. Sin embargo, para el individuo e del ejemplo, puede ser útil saber que de las personas que están en las mismas condiciones (los que tienen VIH), una proporción de 0.61 (61\%) han desarrollado el sida. Para él específicamente, no corresponde a una probabilidad formal sino a una frecuencia relativa calculada con datos de otras personas. Para su caso, nadie está interesado en extraer aleatoriamente una persona con VIH para ver si tiene sida. Pero la frecuencia relativa resume el aprendizaje a partir de experiencias ajenas, que también es valioso.

Un ejemplo famoso es el de la tragedia del Challenger. Los documentos históricos muestran que al menos uno de los investigadores tenía conciencia de que el riesgo era real, que dio a conocer su preocupación, pero que la interpretación que se dio a los estudios de probabilidad determinó que los resultados no eran concluyentes y que, por votación, se autorizó el lanzamiento. Si la tragedia no hubiera ocurrido, seguramente le hubieran dicho que sus dudas eran infundadas y los ejemplos de hoy dirían que el lanzamiento fue exitoso a pesar de los altos riesgos señalados por las probabilidades que ahora se calculan. Realmente, lo más probable es que el público ni siquiera tendría ni el problema ni los datos para "resolver" la tragedia a posteriori.

Este ejemplo es muy interesante, porque para su análisis siempre se han considerado factores que tenían relaciones causales directas con el desenlace y no con información de experiencias ajenas al caso estudiado. Se trataba de una probabilidad propia (objetiva) de un evento irrepetible. En el momento se hicieron cálculos que fueron considerados por unos como suficientemente preocupantes, pero no concluyentes por la mayoría. Posteriormente se hicieron otros cálculos mediante modelos lineales generalizados que no dejarían dudas al respecto. También se hicieron otros 
cálculos basados en modelos bayesianos que dejarían en ridículo a quien quisiera manifestar alguna duda. Sin embargo, los cálculos posteriores a la tragedia son puramente académicos o, si se quiere, especulativos, porque en el momento real, cuando se tomaron las decisiones, no se tenía esa información sobre la mesa. Lo único que nos dicen realmente, es que hoy disponemos de mejores herramientas de análisis.

En el momento histórico, lo disponible era un resultado que dejaba un margen demasiado grande para la subjetividad. Uno de los ingenieros prefirió advertir, por su experiencia y conocimiento personales, es decir, subjetivamente, que el riesgo era suficientemente grande como para aplazar el lanzamiento. Los demás consideraron, también subjetivamente, que los resultados no eran concluyentes y votaron por el lanzamiento. Fue una lucha entre subjetividades donde ganó la equivocada.

A la luz de los resultados, hoy podemos decir que la mejor herramienta para el caso es la bayesiana. Pero, ¿qué nos garantiza que la probabilidad calculada es la correcta? Como el resultado se conoce, entonces podríamos decir que el método que arroje el resultado más cercano de 1 es el mejor. Pero si la tragedia no hubiera ocurrido ¿habría sido considerado como el peor?

Para los eventos irrepetibles, es posible que los mejores cálculos sean los más extremos, es decir, los más cercanos de 0 o de 1, pues así nos dejan con menor margen de incertidumbre. Es claro que no determinan el resultado, pero es ahí donde adquieren el significado de probabilidades. El concepto de entropía permitiría generalizar el criterio cuando los resultados no sean dicotómicos.

Una experiencia familiar ayuda a ilustrar algo más nuestro tema: un pequeño sobrino, gravemente desnutrido y desidratado, fue diagnosticado de estenosis pilórica. El médico advirtió la necesidad urgente de una cirugía. Al preguntarle por los riesgos de muerte durante la operación, respondió que en la clínica habían practicado no menos de 10000 cirugías de este tipo y que no más de 5 niños habían fallecido. Formalmente, la proporción 0.0005 no era una probabilidad de muerte para el niño; pero para el equipo médico sí era un indicador de que sabían hacer las cosas bien. Igual que la probabilidad, este indicador fue un factor determinante para tomar la decisión de autorizar la cirugía. Mi sobrino sobrevivió y hoy ya tiene más de 22 años. ¿Fué la probabilidad la que lo salvó? ¿Cómo estaría interpretando las cosas si hubiera muerto? ¿Cuál decisión hubiera tomado si alguien se me acerca y me dice "cuidado, este médico solo ha practicado 5 cirugías y todos los niños se le han muerto"?

Personalmente, no tengo el afán de interpretar cada fracción entre 0 y 1 como una probabilidad, a menos que tenga los contextos que me convenzan de hacerlo. En muchos casos se dispone de datos acerca de proporciones que se pueden tomar como indicadores de algún tipo de riesgo, pero que en esencia no son probabilidades. En particular, los hechos cumplidos tienen probabilidad 1 de haber ocurrido. Todo cálculo que dé otro valor solo tiene validez en un mundo especulativo. 


\section{Bunge y la subjetividad}

Bunge (2012) destaca que, a lo largo de la historia, el estancamiento de la medicina se ha relacionado con el dominio de criterios atribuidos a dones o a poderes personales adquiridos por artes mágicas o directamente de divinidades. Su desarrollo auténtico solo se ha dado cuando se han logrado identificar relaciones de las enfermedades con alteraciones físicas en determinadas partes del cuerpo. Los avances se aceleraron cuando se logró entender que las actividades físicas, emocionales o intelectuales del hombre estaban estrechamente relacionadas con uno o varios de sus órganos. En la medida en que estas relaciones se han podido comprobar, ha mejorado la capacidad de diagnóstico y se han promovido mejores opciones de tratamientos y de cuidados preventivos.

La medicina ha avanzado en la medida en que se ha dejado de lado el uso de criterios subjetivos y se lo ha reemplazado por el del conocimiento de relaciones objetivas y comprobables. Podríamos agregar que estos progresos también se han dado en la medida en que los conocimientos adquiridos se han podido enseñar y compartir con un espíritu abierto a la crítica.

Del escrito de Bunge entiendo lo importante que es distinguir el uso del conocimiento previo objetivo del manejo de supuestos caprichosos e infundados y de los elaborados a conveniencia de resultados intencionales. Caer en estos últimos equivale a regresar a los dictámenes de "neochamanes" con toda la credibilidad que les daría la utilización ingenua o incluso cómplice de procedimientos estadísticos de cualquier índole.

No siempre el respaldo de datos como fuente de información previa es sinónimo de objetividad. La misma relación con los datos es frágil, tal como lo muestra con el ejemplo de Durkheim (p. 96) sobre las menores tasas de suicidio en los católicos. El investigador debe analizar cuidadosamente la calidad de los datos y los entornos que rodearon su creación antes de utilizarlos como base o respaldo para sus estudios. Desgraciadamente, muchas veces se descubren sus problemas solo cuando han generado controversia.

Bunge ataca decididamente la subjetividad y arremete furiosamente contra los bayesianos cuando observa que le abren la puerta. Ve inminente el regreso a las decisiones fuertemente influenciadas por opiniones no comprobables de personas que han ganado algún reconocimiento, pero que no corresponden a la realidad. Lo ve como una amenaza al progreso de la ciencia y especialmente de la medicina. Además, agravada por el respaldo de una disciplina que gana vertiginosamente credibilidad en la sociedad científica.

¿Quién de nosotros no ha visto publicaciones o al menos comunicados de prensa donde se anuncia el éxito de una dieta o de un tratamiento para combatir una enfermedad, descalificado pocos meses después porque se encuentra que el único respaldo del estudio eran unos resultados estadísticos donde el $80 \%$ de las personas que manifestaban haberse recuperado recordaban haber consumido alguna fruta? Ningún estudio sobre la relación entre los componentes de la fruta y la enfermedad. 
Ningún estudio realmente médico. Solo una intuición personal "respaldada" por unos resultados numéricos obtenidos con métodos estadísticos también oscuros o provenientes de una "minería de datos" mal entendida.

El teórico demuestra que los métodos están bien diseñados y desarrollados y en su ataque se equivoca Bunge. Pero es en las aplicaciones en las que la condición humana le da la razón al filósofo. Ya el ejemplo del Challenger pone en evidencia que hasta en esferas de alta calidad científica la subjetividad o incluso determinados intereses pueden cegar la razón.

\section{Interpretación de un mensaje filosófico}

A mi modo de ver, el clamor de Mario Bunge es por la responsabilidad social del médico. Seguramente en la atención particular a sus pacientes se verá con frecuencia en la necesidad de acudir a su experiencia personal o a la de sus colegas para proponer diagnósticos o soluciones. Pero para generar y divulgar conocimiento, deberá desterrar la subjetividad y buscar explicaciones objetivas que lo acerquen a la realidad de la naturaleza de las enfermedades, de su diagnóstico, de su tratamiento, de sus causas y de su posible prevención. Estoy de acuerdo con Bunge en que "la estadística es indispensable para estudiar y manipular poblaciones de todo tipo [...]. Pero no suministra conocimiento sustantivo. Ni puede hacerlo, porque no se ocupa de las cosas mismas sino de datos acerca de ellas".

También es un llamado a la responsabilidad social del estadístico, cualquiera sea su orientación metodológica. Todos sabemos de la importancia de la estadística para la toma de decisiones y para el desarrollo de casi todas las disciplinas; que su aplicación cuidadosa ofrece una ventana de transparencia y de rigor científico difícil de reemplazar. En medio del ataque de Bunge, percibo una defensa de mayor dimensión: proteger a la estadística, bayesiana o no, del uso irracional o intencionado de la subjetividad.

Recibido: 17 de agosto de 2013 Aceptado: 30 de agosto de 2013

\section{Referencias}

Bunge, M. (2003), Cápsulas, Gedisa, Barcelona.

Bunge, M. (2012), Filosofía para médicos, Gedisa, Barcelona. 